\title{
Satellite Image Enhancement for Fusion of Multispectral and Panchromatic Images
}

\author{
Kavana A M $\mathbf{M}^{1}$, Dr. Mohamed Rafi ${ }^{2}$ \\ ${ }^{1}$ PG Student, Computer Science and Engineering, UBDTCE Davanagere, Karnataka, India \\ ${ }^{2}$ Professor, Computer Science and Engineering, UBDTCE Davanagere, Karnataka, India
}

\begin{abstract}
Pan-sharpening is the way toward enhancing spatial determination of multi-phantom (MS) satellite pictures utilizing the spatial subtle elements of a high determination Panchromatic (PAN) picture. The combination of multispectral (MS) and panchromatic $(P A N)$ pictures is a valuable procedure for upgrading the spatial nature of low-determination MS pictures. Picture combination is the procedure by which input pictures are melded so as to increment the quality. The information pictures must be the pictures of a similar scene with various quality measures. The nature of the yield picture will be superior to anything any of the information pictures. To talk about the use of this technique to a famous remote detecting application called container honing, which comprises of the combination of a low determination multispectral picture and a high - determination panchromatic picture. The principle techniques for the picture combination include straightforward picture combination, pyramid based picture combination and wavelet based picture combination and to centers around Four informational collections by the Pleiades, Worldview-2, Icons, and Geoeye-1 satellites are utilized for the execution evaluation, affirming the viability correlation in light of the nature of the yield picture with and without Discrete Wavelet Transform. Higher visual quality is accomplished by performing DWT based addition. It likewise talks about the diverse quality measurements that can be utilized to evaluate the nature of the yield picture
\end{abstract}

Keywords: Image fusion, Panchromatic images, Multispectral images, PCA, Wavelet

\section{Introduction}

Image processing is increasing more significance in the ranges of science and innovation. It constitutes a promising territory of look into because of constantly developing significance of logical perception in different applications. The need of better execution in the picture preparing expanded the requests on computational efficiencies. Different options are accessible to enhance the execution of picture preparing utilizing specific structures. Picture combination is a procedure of blending the significant data from a few info pictures into a solitary picture. It is widely utilized as a part of picture handling applications like administration of normal assets, remote detecting, protection and medicinal imaging. Different combination strategies are accessible to enhance the nature of melded picture. In remote detecting applications, satellites give the data of the expansive ranges of the planet. To address the issues of a few remote detecting applications, for example, climate, meteorological and ecological, the remote detecting framework offers spatial, ghastly, radiometric and worldly resolutions. By and large, satellites take different pictures from various frequencies in the visual and non-visual extents called as monochrome pictures. In view of the recurrence extend every monochrome picture contains different data about the question. Each monochrome picture is spoken to as a band and an accumulation of these groups of a similar scene acquired by a sensor is called multispectral picture (MS). When all is said in done, a MS picture contains three groups (Red, Green and Blue). The blend of these three groups creates a shading picture. Satellites more often than not give a panchromatic (PAN) picture alongside MS picture. A PAN picture alludes to a dim scale picture that contains the information with an extensive variety of wavelengths from the obvious to the warm infrared.
Asset shortage and ecological debasement influence human security in various measurements, as they likewise assume a part in producing and compounding fierce clash [120]. Fig. 1.1 demonstrates ASTER (Advance Space borne Thermal Emission and Reflection Radiometer) imaging of a locale in Rondonia, Brazil.1

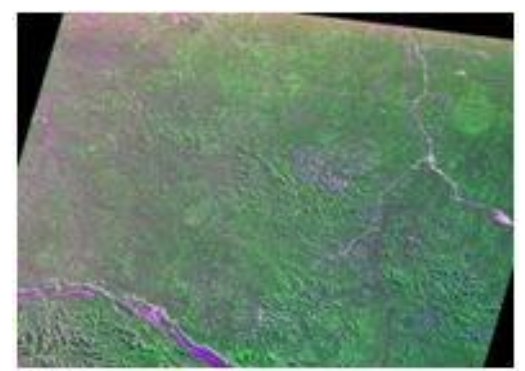

Figure 1: Aster picture of a locale in Rondonia, Brazil.

In the ASTER false shading composite (joining near infrared, red, and green groups), tropical rainforest compares to splendid red, dark colored highlights deforested land, and dark and dim demonstrate as of late consumed ranges.

\section{Prior Work}

\subsection{Nonlinear Decomposition Schemes.}

Generally, the fusion can be divided into two distinct parts.

- Up scaling of the MS images, so that the up scaled MS images have the same size as the PAN image.

- The process of fusion, which may be achieved either by the addition of the high-frequency content of the PAN image to the MS image or by substitution of the intensity image by the PAN image in case of the IHS algorithm. 


\section{International Journal of Science and Research (IJSR) \\ ISSN (Online): 2319-7064}

Index Copernicus Value (2015): 78.96 | Impact Factor (2015): 6.391

Detailed geographic data is a key figure choice making forms amid evacuee alleviation operations. The inevitable business high spatial determination (VHSR) satellite sensors will be fit for gaining multispectral (MS) pictures at spatial resolutions of $1 \mathrm{~m}$ (panchromatic) and $4 \mathrm{~m}$ (multispectral) of evacuee camps and their condition.

This work shows how evacuee camp condition, range also, populace can be broke down utilizing a VHSR MS satellite sensor picture from the Russian KVR-1000 sensor. The VHSR MS picture was observed to be reasonable for mapping the displaced person camp condition and range. A factually huge straight connection between camp range and populace was decided. The procedure comprises of applying the POHMT to multispectral pictures to identify potential building areas. The measure of the organizing component (SE) was characterized through the morphological top-cap by remaking change. To expel unessential areas, vegetation ranges were covered utilizing the standardized contrast vegetation file (NDVI), and the distinguished areas were checked by the closeness of shadows. The fruitful acknowledgment of articles with the HMT, what's more, thusly POHMT, relies on upon the likelihood of characterizing layouts that relate to the setups of protest/neighborhood. The assortment of shapes and sizes of structures would include the meaning of extensive quantities of formats.

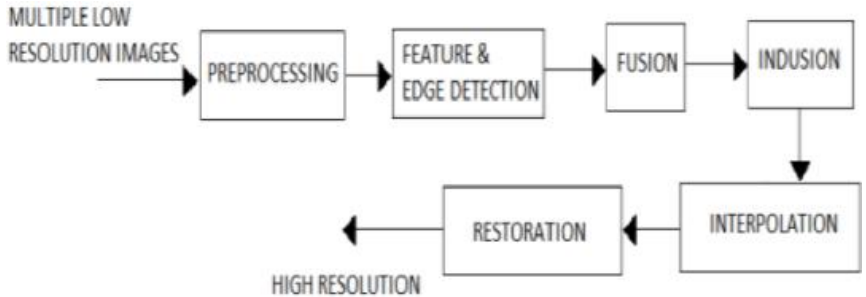

Figure 2: Block diagram of SFIM based image fusion.

To stay away from this and apply the POHMT in an unsupervised way, we made a few speculations. As little structures have a zone of around, we settled a consistent size for the FGSE as indicated by the spatial determination of the pictures utilized pixels for the skillet honed Mikonos picture, and pixels for the Geo Eye and WorldView-2 pictures. Along these lines, we guaranteed that the FGSE would not surpass the span of little structures, and that the disintegration and enlargement could be characterized over them. Expanding the span of the FGSE will miss structures littler than this size. For instance, on the off chance that we utilized a pixel window for the Geo Eye picture, structures littler than could have been missed.

\subsection{Indusion}

In this section, another combination strategy is created in light of Enlistment. Since the procedure is gotten from Induction and consolidates combination, it is named "Indusion." We can rework (3.2) as

$$
\mathrm{K}=\mathrm{J}-[[\mathrm{J} * \mathrm{R}] \downarrow \mathrm{a}] \uparrow \mathrm{a} * \mathrm{~A}+[\mathrm{I}] \uparrow \mathrm{a} * \mathrm{~A}
$$

The underlined some portion of the condition speaks to the high recurrence data, spatial edges, while the rest speaks to the low-recurrence part of the picture. In (3.3), J is the upscale variant of the underlying picture I. In remotedetecting picture combination, the thought is to separate the high frequency data of the PAN picture and add it to the upscale low-determination MS picture. The above condition proposes that the high-recurrence data from $\mathrm{J}$ can be added to the upscale variant of the underlying low-determination picture I. The thought of Indusion is to supplant picture $\mathrm{J}$ by the PAN picture since we need the high-recurrence data of the PAN picture to be added to the upscale MS picture. The changed condition moves toward becoming

$$
\mathrm{K}=\mathrm{PAN}-[[\mathrm{PAN} * \mathrm{R}] \downarrow \mathrm{a}] \uparrow \mathrm{a} * \mathrm{~A}+[\mathrm{I}] \uparrow \mathrm{a} * \mathrm{~A}
$$

The Indusion calculation was tried on genuine Quick fowl satellite pictures and recreated Pleiades pictures. Since the proportion between the PAN and MS pictures given by them is 1: 4 , the procedure is partitioned into two phases, each with a scaling element

\section{Proposed Method}

The proposed scaling calculation is a region pixel scaling calculation comprising of a preprocessor, channel, discrete wavelet change based insertion, morphological administrator, insertion and PCA based combination is appeared in Fig. 4.1. Preprocessor changes over the first picture to dim scale esteems. To accomplish higher visual quality, discrete wavelet change based introduction is done first and afterward addition. DWT depends on sub band coding, which partitions the picture into four recurrence quadrants and afterward addition is conveyed out to all the four quadrants independently which diminishes the curios. Insertion is a productive strategy in which the flat and vertical insertion with closest 16 pixels is done, which enhances the determination of the picture. At long last the panchromatic picture intertwined with the multiresolution picture and at long last gets the high determination picture. Here it is proposed to embrace a plan that consolidate PCA with Discrete Wavelet Change to create a prevalent come about than utilizing PCA strategy alone or DWT strategy alone. However the exchange off is higher multifaceted nature and cost.

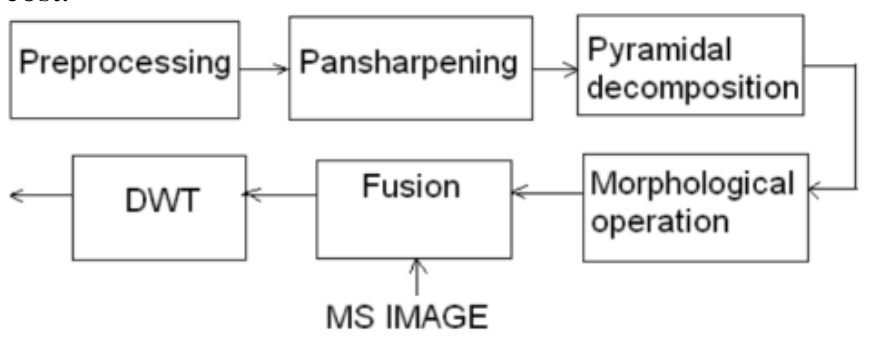

Figure 3: Block diagram for Proposed Scheme

In spatial space procedures, we straightforwardly manage the picture pixels. The pixel esteems are controlled to accomplish wanted result. In recurrence space strategies the picture is first moved into recurrence space. It implies that the Fourier Change of the picture is registered first. All the Fusion operations are performed on the Fourier change of the picture and afterward the Inverse Fourier change is performed to get the resultant picture. In PC vision,

\section{Volume 6 Issue 7, July 2017 www.ijsr.net}




\section{International Journal of Science and Research (IJSR) \\ ISSN (Online): 2319-7064}

Index Copernicus Value (2015): 78.96 | Impact Factor (2015): 6.391

Multisensory Image combination is the way toward consolidating pertinent data from at least two pictures into a solitary picture. The subsequent picture will be more useful than any of the information pictures.

Phantom mutilation turns into a negative component while we go for additionally preparing, for example, order issue. Spatial mutilation can be exceptionally all around taken care of by recurrence area approaches on picture combination. The multi determination investigation has turned into an extremely helpful apparatus for dissecting remote detecting pictures. The discrete wavelet change has turned into an extremely valuable device for combination. Some other combination techniques are likewise there, for example, Laplacian-pyramid based, Curvelet change based and so forth. These techniques demonstrate a superior execution in spatial and ghostly nature of the melded picture contrasted with other spatial techniques for combination. There are different techniques that have been produced to perform picture combination. Some well-known image fusion methods are listed below:-

(1) Intensity-hue-saturation (IHS) transform based fusion

(2) Principal component analysis (PCA) based fusion

(3) Multi scale transform based fusion:-

(a) High-pass filtering method

(b) Pyramid method:-

(i) Gaussian pyramid

(ii) Laplacian Pyramid

(iii) Gradient pyramid

(iv) Morphological pyramid

(v) Ratio of low pass pyramid

(1) Panchromatic pictures - A picture gathered in the expansive visual wavelength extend yet rendered in highly contrasting.

(2)Multispectral pictures - Images optically procured in additional than one unearthly or wavelength interim. Every individual picture is for the most part of the same physical range and scale however of an alternate phantom band. The SPOT PAN satellite gives high determination (10m pixel) panchromatic information, while the LANDSAT TM satellite gives low determination (30m pixel) multispectral pictures. Picture combination endeavors to blend these pictures and create a solitary high determination multispectral picture. The standard blending techniques for picture combination depend on RedGreen-Blue (RGB) to Intensity-Hue-Saturation (IHS) change.

The typical strides required in satellite picture combination are as per the following: Modify the panchromatic picture with regard to the multispectral picture. This is typically performed by histogram coordinating of the panchromatic picture with Intensity part of the multispectral pictures as reference. Supplant the power part by the panchromatic picture and perform backwards change to get a high determination multispectral picture.

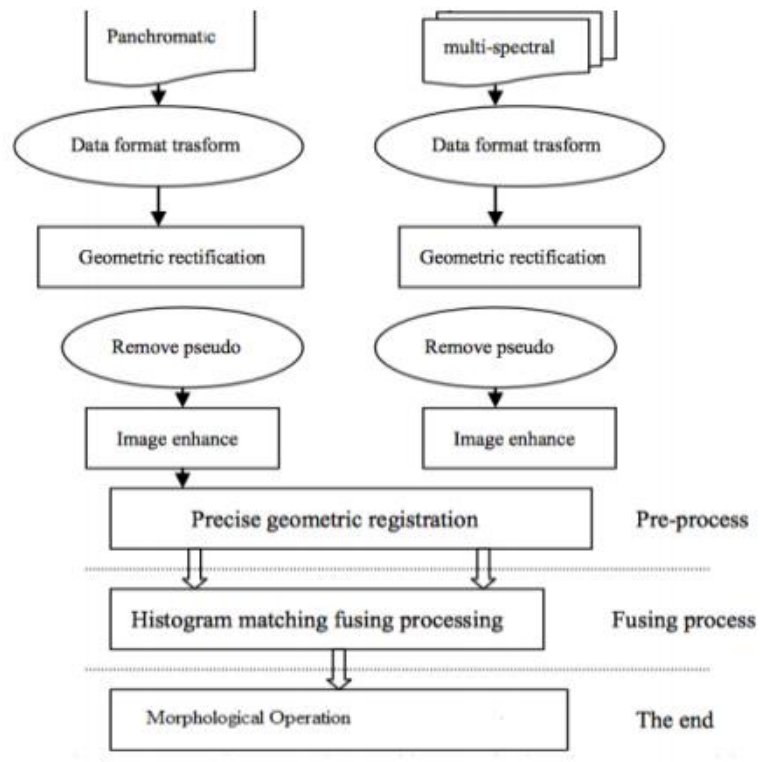

Figure 4: Distortion

\subsection{Discrete Wavelet Transform}

This wavelet change has increased across the board acknowledgment in flag preparing and picture pressure. The DWT has been presented as an exceedingly proficient and adaptable strategy for disintegration of signs. In DWT, flag vitality concentrates to particular wavelet coefficients. Here a period scale portrayal of the advanced flag is gotten utilizing computerized sifting procedures. The flag to be dissected is gone through channels with various cut-off frequencies at various scales. It is simple to execute and diminishes computational time and assets required. DWT processor changes the spatial area pixels into recurrence space data that are spoken to in numerous sub groups speaking to various time scales and recurrence focuses.

\subsection{Principal Component Analysis (PCA)}

PCA is a scientific device which changes various corresponded factors into various uncorrelated factors. The PCA is utilized widely in picture pressure and picture characterization. The PCA includes a scientific method that changes various corresponded factors into various uncorrelated factors called important segments. It figures a reduced and ideal portrayal of the informational collection. The main chief segment represents as a significant part of the change in the information as could be expected under the circumstances and each succeeding part represents however much of the rest of the change as could be expected. To start with essential segment is taken to be along the course with the most extreme fluctuation. The second key segment is obliged to lie in the subspace opposite of the first. Inside this Subspace, this segment focuses the course of most extreme fluctuation. The third key segment is taken in the most extreme change course in the subspace opposite to the initial two et cetera. The PCA is likewise called as KarhunenLoève change or the Hotelling change. The PCA does not have a settled arrangement of premise vectors like FFT, DCT and wavelet and so forth. What's more, its premise vectors rely on upon the informational index. Perform histogram 


\section{International Journal of Science and Research (IJSR) \\ ISSN (Online): 2319-7064}

Index Copernicus Value (2015): 78.96 | Impact Factor (2015): 6.391

coordinate process amongst PAN and MS pictures to get three new PAN pictures. Utilize the Wavelet Transform to decay new PAN pictures and distinctive groups of MS picture. Include the detail pictures of the decayed PAN picture at various levels to the comparing subtle elements of various groups in the MS picture what's more, acquire the new points of interest segment in the distinctive groups of the MS picture. Perform Inverse Wavelet Transform on the groups of MS pictures separately and get the combined picture. At the point when the quantity of pictures to be melded is higher than three i.e. combination of multidimensional information, key part investigation (PCA) a factual apparatus (which is otherwise called KarhunenLo'eve change) is utilized for dimensionality lessening. In the most regular comprehension, PCA is an information pressure strategy changing the inter correlated information into another set of uncorrelated parts $(\mathrm{PC} 1, \mathrm{PC} 2, \ldots, \mathrm{PCn}$, where $\mathrm{n}$ is the quantity of information multispectral groups) which is frequently more interpretable than the source information. It processes the premise vectors by breaking down the course of most extreme information fluctuation and ventures the information onto them.

$$
\begin{aligned}
& D=\left[\begin{array}{ccc}
D_{1} & \cdots & 0 \\
\vdots & \vdots & \vdots \\
0 & \cdots & D_{n}
\end{array}\right] \\
& E * \operatorname{Cov} * E^{T}=D
\end{aligned}
$$

Where, $\mathrm{E}=$ matrix of eigenvectors, $\mathrm{Cov}=$ covariance matrix, $\mathrm{T}=$ transposition function and $\mathrm{D}=$ diagonal matrix of eigen values in which all non-diagonal elements are zeros and nonzero elements are ordered from greatest to least.

\subsection{Quantitative Measures}

In Mathematical displaying, quantitative measure is alluring. Quantitative measures are utilized to foresee the apparent picture quality. In this review, quality evaluation utilizing commotion based measures are utilized to assess the commotion of the intertwined picture by contrasting with its unique MS picture. The accompanying ideal clamor based measures are executed to judge the execution of the above examined combination techniques. Entropy is used to measure the amount of data contained in the melded picture. A greater esteem demonstrates great combination comes about.

\subsection{Morphological Operation}

Morphology is a wide arrangement of picture preparing operations that handle pictures in view of shapes. Morphological operations apply an organizing component to an information picture, making an yield picture of a similar size. In a morphological operation, the estimation of every pixel in the yield picture depends on a examination of the comparing pixel in the info picture with its neighbors.

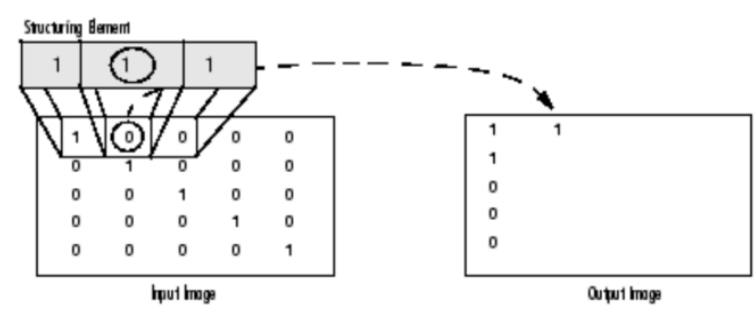

Figure 5: Morphological Operation

By picking the size and state of the area, a morphological operation that is touchy to particular shapes in the information picture can be built. The most essential morphological operations are expansion and disintegration. Widening adds pixels to the limits of items in a picture, while Disintegration expels pixels on protest limits. The quantity of pixels included or expelled from the items in a picture depends on the size and state of the organizing component used to handle the picture. In the morphological widening and disintegration operations, the condition of any given pixel in the yield picture is controlled by applying a manage to the relating pixel and its neighbors in the info picture. The manage used to prepare the pixels characterizes the operation as enlargement or disintegration. A fundamental some portion of the enlargement and disintegration operations is the organizing component that is utilized to test the info picture. An organizing component is a framework comprising of just 0 's and 1's that can have any subjective shape and size. The pixels with estimations of 1 characterize the area.

\section{Conclusion and Future Work}

The investigation of the picture combination in view of Discrete Wavelet Change, on the premise of concentrate the general techniques, proposes another idea: distinctive combination principles will be utilized as a part of low recurrence picture and high recurrence picture. The novel approach of consolidating the DWT and PCA techniques for picture combination gave better outcomes in handling of satellite symbolism. The results are urging to try different things with blend of existing techniques to get more exact outcomes. What's to come step is to apply the weighted combination method on multi sensor picture to distinguish objects.

\section{References}

[1] S. Mayunga, Y. Zhang, and D. Coleman, "Semi-automatic building extraction utilizing Quickbird imagery," in Proc. ISPRS Workshop CMRT, 2005, pp. 131-136.

[2] A. Carleer, O. Debeir, and E. Wolff, "Assessment of very high spatial resolution satellite image segmentations," Photogramm. Eng. Remote Sens., vol. 71, no. 11, pp. 1285-1294, Nov. 2005

[3] P. Gamba, F. Dell'Acqua, G. Lisini, and G. Trianni, "Improved VHR urban area mapping exploiting object boundaries," IEEE Trans. Geosci. Remote Sens., vol. 45, no. 8, pp. 2676-2682, Aug. 2007.

[4] Y. Wei, Z. Zhao, and J. Song, "Urban building extraction from highresolution satellite panchromatic image using clustering and edge detection," in Proc. IEEE Int. Geosci. 
Remote Sens. Symp. (IGARSS '04), vol. 3, 2004, pp. 2008-2010.

[5] H. Xu and P. Li, "Urban land cover classification from very high resolution imagery using spectral and invariant moment shape information," Can. J. Remote Sens., vol. 36, no. 3, pp. 248-260, Jun. 2010.

[6] B. Sirmaçek and C. Unsalan, "Building detection using local Gabor features in very high resolution satellite images," in Proc. IEEE 4th Int. Conf. Recent Adv. Space Technol. RAST, Jun. 2009, pp. 283-286.

[7] J. Peng, D. Zhang, and Y. Liu, "An improved snake model for building detection from urban aerial images," Pattern Recognit. Lett., vol. 26, no. 5, pp. 587 595, Apr. 2005. of the IEEE Congress on 